\title{
Comparative Analysis Between Thoracic Spinal Cord and Sacral Neuromodulation in a Rat Spinal Cord Injury Model: A Preliminary Report of a Rat Spinal Cord Stimulation Model
}

\author{
Seung-Jae Hyun ${ }^{1}$, Chang-Hyun Lee', Ji Woong Kwon", Cheol-Yong Yoon², \\ Jae-Young Lim ${ }^{3}$, Ki-Jeong Kim', Tae-Ahn Jahng ${ }^{1}$, Hyun-Jib Kim ${ }^{1}$ \\ Departments of Neurosurgery ${ }^{1}$, Urology ${ }^{2}$, and Rehabilitation Medicine, Seoul National University Bundang Hospital, Seoul \\ National University College of Medicine, Seongnam, Korea
}

Objective: The purpose of this study is to compare a neuroprotective effect of thoracic cord neuromodulation to that of sacral nerve neuromodulation in rat thoracic spinal cord injury $(\mathrm{SCl})$ model.

Methods: Twenty female Sprague Dawley rats were randomly divided into 4 groups: the normal control group ( $n=5), \mathrm{SCl}$ with sham stimulation group $(\mathrm{SCl}, \mathrm{n}=5), \mathrm{SCl}$ with electrical stimulation at thoracic spinal cord $(\mathrm{SCl}+\mathrm{TES}, \mathrm{n}=5)$, and $\mathrm{SCl}$ with electrical stimulation at sacral nerve $(\mathrm{SCl}+\mathrm{SES}, \mathrm{n}=5)$. Spinal cord was injured by an impactor which dropped from $25 \mathrm{~mm}$ height. Electrical stimulation was performed by the following protocol: pulse duration, $0.1 \mathrm{~ms}$; frequency, $20 \mathrm{~Hz}$; stimulation time, 30 minutes; and stimulation duration at thoracic epidural space and S2 or 3 neural foramina for 4 weeks. Locomotor function, urodynamic study, muscle weights, and fiber cross sectional area (CSA) were investigated.

Results: All rats of the SCI + TES group expired within 3 days after the injury. The locomotor function of all survived rats improved over time but there was no significant difference between the SCl and the SCl + SES group. All rats experienced urinary retention after the injury and recovered self-voiding after 3-9 days. Voiding contraction interval was $25.5 \pm 7.5$ minutes in the SCl group, $16.5 \pm 5.3$ minutes in the SCI+SES group, and 12.5 \pm 4.2 minutes in the control group. The recovery of voiding contraction interval was significant in the $\mathrm{SCl}+\mathrm{SES}$ group comparing to the $\mathrm{SCl}$ group $(\mathrm{p}<0.05)$. Muscle weight and CSA were slightly greater in the SCl + SES than in the SCl group, but the difference was not significant.

Conclusion: We failed to establish a rat spinal cord stimulation model. However, sacral neuromodulation have a therapeutic potential to improve neurogenic bladder and muscle atrophy.

Key Words: Electrical stimulation $\cdot$ Spinal cord injury $\cdot$ Neuroprotection $\cdot$ Neuromodulation

\section{INTRODUCTION}

The spinal cord injury (SCI) often leads to serious neurological sequelae and various complications such as neurogenic bladder, lower extremities muscle atrophy, etc. Electrostimulation on sacral nerve roots is currently being tried with some promising results as a treatment for a wide spectrum of voiding dysfunctions. Besides, several investigators reported addi-

- Received: December 16, 2012 - Revised: February 23, 2013

- Accepted: March 13, 2013

Corresponding Author: Seung-Jae Hyun, MD, PhD

Department of Neurosurgery, Spine Center

Seoul National University Bundang Hospital, Seoul National University College

of Medicine, 300 Gumi-dong, Bundang-gu, Seongnam-si, Gyeonggi-do

463-707, Korea

Tel: +82-31-787-7164, Fax: +82-31-787-4059

E-mail: neurospine@snubh.org tional effects of the sacral nerve stimulation such as change of spasticity and spasm, increased motility of intestinal tract and defecation, and improvement of locomotor function in experimental and clinical studies, ${ }^{3,6,11,12,14)}$. Furthermore, there is a case report about additional improvement of lower extremity motor ${ }^{6}$. They reported that one patient with a long history of progressive spinal multiple sclerosis was able to stand and transfer, but not walk, before the sacral nerve stimulation $^{6}$. Another paraplegic patient was showed improvement of motor power slowly, and after 2 years the patient could stand as confident as before ${ }^{6}$.

Our institution has established a rat sacral neuromodulation model after $\mathrm{SCI}^{12}$. The purpose of this study is (1) to establish a rat thoracic spinal cord stimulation model and (2) to compare effects of thoracic cord neuromodulation to that of sacral nerve neuromodulation on locomotor function, bladder and lower extremities muscles. 


\section{MATERIALS AND METHODS}

Twenty Sprague Dawley rats of 6 months old female, weighing 200 to $250 \mathrm{gm}$, were randomly divided into 4 groups: a normal control group $(\mathrm{n}=5)$, SCI with sham stimulation group (SCI, $\mathrm{n}=5)$, and $\mathrm{SCI}$ with electrical stimulation at thoracic spinal cord (SCI + TES, $n=5)$, and SCI with electrical stimulation at sacral nerve (SCI + SES, $n=5)$. The control group included rats that were both uninjured and unstimulated. The SCI group included rats that were injured with electrodes implanted but did not receive stimulation. The SCI + TES and SCI + SES group included rats that received epidural electrical stimulation at the thoracic spinal cord or S2/S3 nerve root using needle electrode, respectively. Rats in the each group were kept in separate cages under the same living conditions for a week before receiving SCI.

\section{Spinal Cord Injury and Electrical Stimulation}

Rats were deprived of food and water for 12 hours before SCI. The animals were anesthetized with an intramuscular injection of Zoletil $15 \mathrm{mg}$ and Xylazine $3 \mathrm{mg}$. Under general anesthesia, rats were placed in a prone position. After routine disinfection, a surgical incision was made through the skin, subcutaneous tissue, and the T8-12 vertebral lamina to the spinal canal. Total laminectomy of T9 or T10 was performed. Rostral and caudal spinous processes were fixed by clamp. The SCIs were induced in rats using an NYU-MASCIS (New York

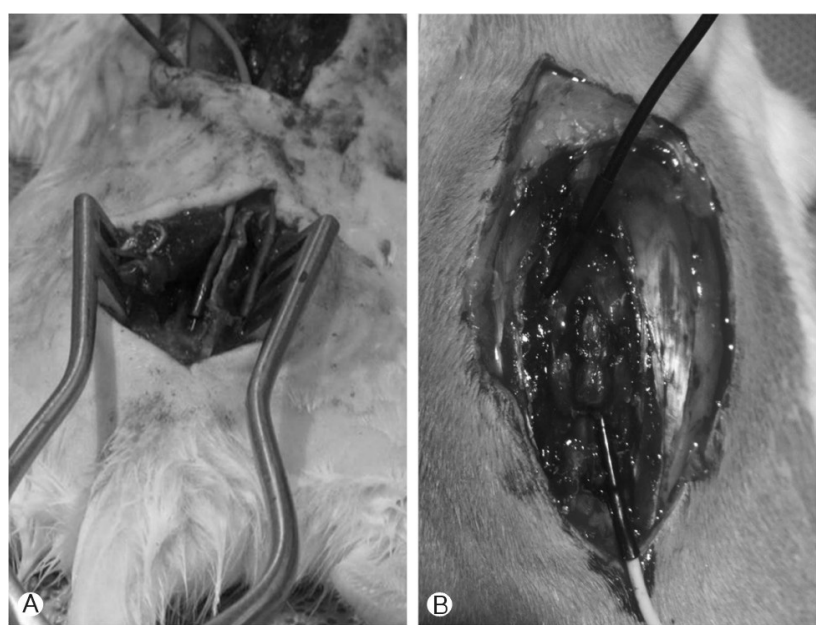

Fig. 1. (A) Exposure of spinal cord at T10-11 by laminectomy. Fine needle electrodes $(0.5 \times 27 \mathrm{G})$ were implanted into S2 foramina. (B) For thoracic spinal cord stimulation, we put the needle electrode on the thoracic epidural space through the laminectomy site. Another electrode was inserted into thoracic paraspinal muscle to earth.
University-Multicenter Animal Spinal Cord Injury Study) impactor. The impactor weighed $10 \mathrm{gm}$ was dropped once from 25 $\mathrm{mm}$ height. The impact height of $25 \mathrm{~mm}$ was considered for severe cord injury based on a prior investigation ${ }^{1)}$.

For sacral neuromodulation, a surgical incision at the S1-3 level was made through the skin, subcutaneous tissue, and the S2-3 vertebral lamina (Fig. 1A). Needle electrodes $(0.5 \times$ $27 \mathrm{G})$ were implanted at bilateral S2 or S3 neural foramina. Wounds were cleaned and sutured with wires inserted through the subcutaneous tissue extruding from the neck. For thoracic spinal cord stimulation, we put the needle electrode on the thoracic epidural space through the laminectomy site (Fig. 1B). The SCI + TES and SCI + SES groups received electrical stimulation 7 days after SCI with the following protocol: stimulation time 30 minutes; pulse duration, $0.1 \mathrm{~ms}$; and frequency, $20 \mathrm{~Hz}$. Epidural low frequency electric stimulation was applied 30 minutes per day for 4 weeks. The electrical stimulation treatment protocol is similar to that previously reported ${ }^{7,9,12,15)}$.

\section{Functional and Histological Outcome}

Post-injury motor behavior is assessed by the Basso, Beattie, and Bresnahan (BBB) locomotor scale method ${ }^{4,5)}$. The BBB score is used for functional recovery and locomotors testing in SCI study. The scale (0-21) represents sequential recovery stages and categorizes combinations of rat joint movement, hindlimb movements, stepping, forelimb and hindlimb coordination, trunk position and stability, paw placement, and tail position. To evaluate the bladder contracting function, urodynamic study using cystometrogram was performed with a serial fashion. All rats were sacrificed at 4 weeks after SCI. Under general anesthesia, the gastrocnemius muscle was harvested, weighed, and stained with hematoxylin and eosin (H\&E) to identify the muscle fiber cross-sectional area (CSA).

\section{RESULTS}

All five rats of the SCI + TES group expired within 3 days after the injury. Three of the five suddenly died after the first stimulation on the thoracic spinal cord. The remaining two also expired after the second spinal cord stimulation. All rats but the SCI + TES group survived through the entire 4 weeks.

Before the injury, all rats showed normal function on the BBB score. All injured rats exhibited a severe BBB score at 1 day after SCI. At day 1 of SCI, the BBB scores of the SCI and the SCI + SES group were $2.8 \pm 0.7$ and $2.7 \pm 0.8$, respectively. The difference between two groups was not significant. During subsequent scoring periods, BBB scores increased and plateaued around 14 days after SCI. The locomotor function im- 
proved significantly at days 3,7 , and 14 after SCI ( 3 days: $7.3 \pm 1.2$ and $7.3 \pm 1.5,7$ days: $13.1 \pm 0.9$ and $13.2 \pm 1.1$, and 14 days: $14.6 \pm 0.8$ and $14.7 \pm 0.6$. The all survived rats improved over time but there was no significant difference at any point in time between SCI and SCI + SES groups.

All rats experienced urinary retention after the injury and recovered self-voiding after 3-9 days. Voiding contraction interval was $25.5 \pm 7.5$ minutes in the SCI group, $16.5 \pm 5.3 \mathrm{mi}-$ nutes in the SCI + SES group, and 12.5 \pm 4.2 minutes in the control (Fig. 2). The recovery of voiding contraction interval was significant in the SCI + SES group comparing to the SCI group $(\mathrm{p}<0.05)$.

The gastrocnemious muscle weight decreased after the SCI, whereas the weight increased by $7.9 \%$ after sacral neuromodulation. The CSA of the muscle was slightly greater in the $\mathrm{SCI}+\mathrm{SES}$ than in the SCI group, however, the difference was not significant. Cross-sectional shape of the muscle fiber was markedly turned into round by SCI. After sacral neuromodulation, the shape was turned into angled again (Fig. 3 and 4).

\section{DISCUSSION}

This study was designed to establish a rat thoracic spinal cord stimulation model and to compare effects of thoracic cord neuromodulation to that of sacral nerve neuromodulation on locomotor function, bladder and lower extremities muscles. However, we failed to accomplish the comparative analysis, because all five rats of the SCI + TES group expired within 3 days after the injury. Three of the five suddenly died after the first stimulation on the thoracic spinal cord. The remaining two also expired after the second spinal cord stimulation. We suggest that the cause of death is associated with epidural spinal cord stimulation in the thoracic spine which is located near the heart. A feasible etiology is cardiac arrest or cardiac arrhythmias.
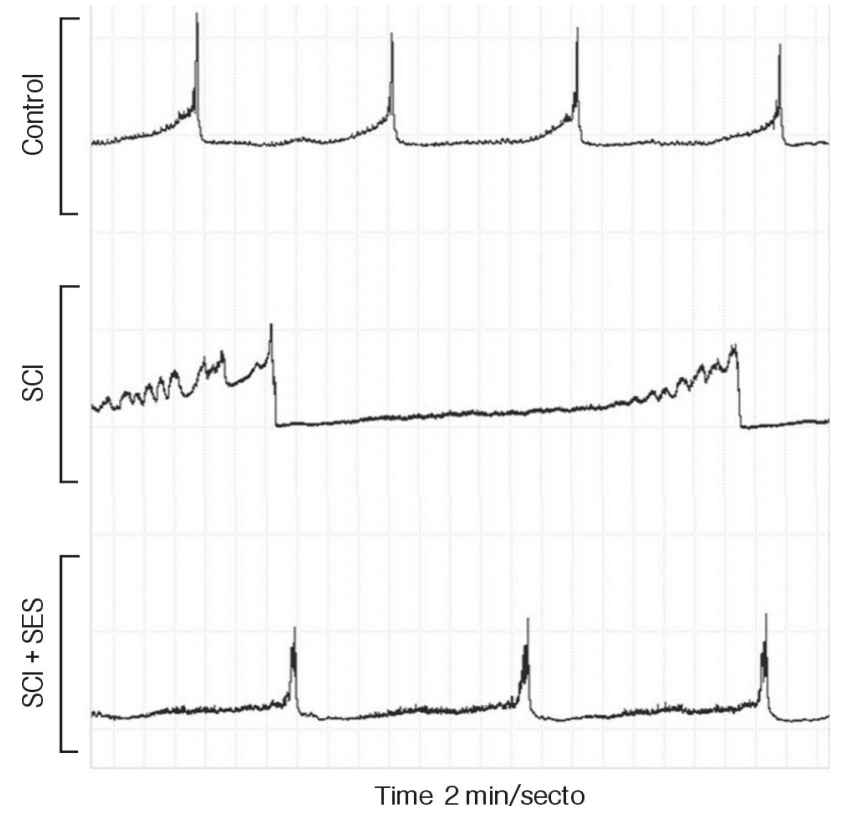

Fig. 2. Representative diagrams of the rat urodynamic study using cystometrogram. Voiding contraction interval was $25.5 \pm 7.5$ minutes in the spinal cord injury $(\mathrm{SCl})$ group, $16.5 \pm 5.3$ minutes in the $\mathrm{SCl}$ with electrical stimulation at sacral nerve group, and 12.5 \pm 4.2 minutes in the control. The shortening of voiding contraction interval means recovery from neurogenic bladder.

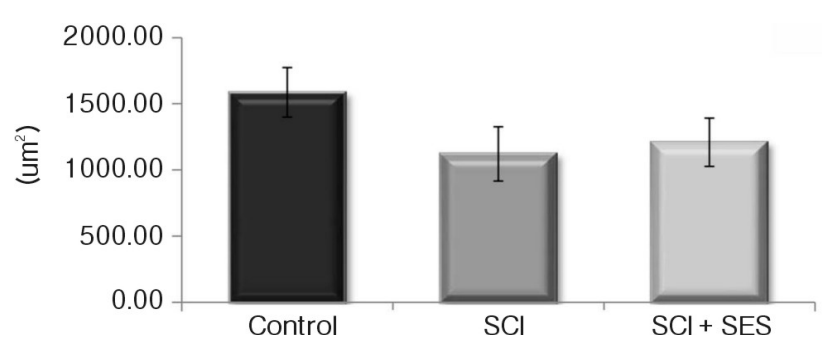

Fig. 3. The change of cross-sectional area (CSA) in gastrocnemious muscle. The CSA of gastrocnemious muscle decreased after the $\mathrm{SCl}$, whereas the weight increased after sacral neuromodulation.

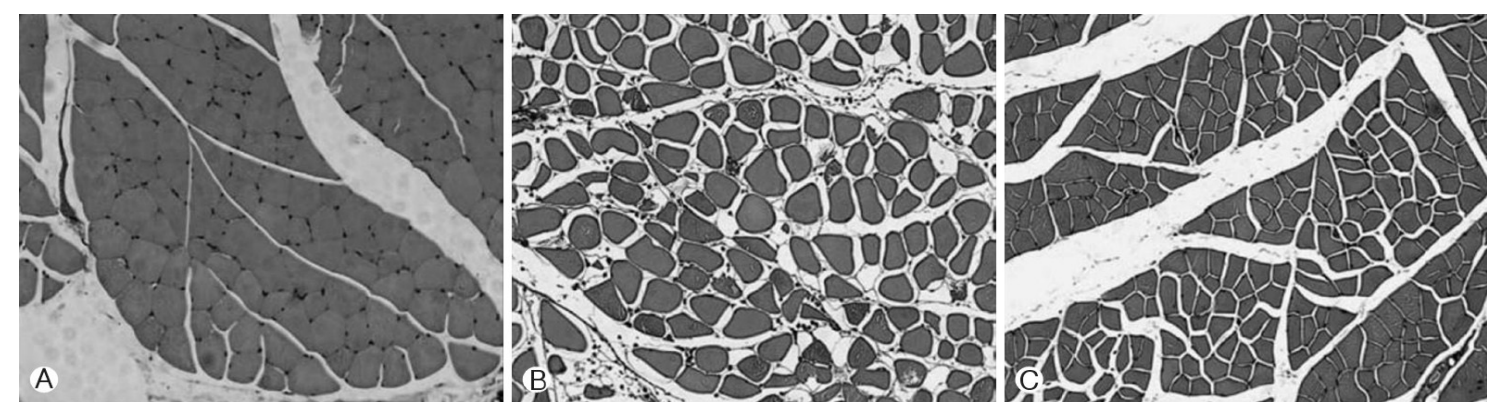

Fig. 4. Representative photomicrographs of hematoxylin and eosin stains in gastrocnemious muscle were used to identify each treatment. (A) control group. (B) spinal cord injury (SCl) group. (C) SCl with electrical stimulation at sacral nerve group. Cross-sectional shape of the muscle fiber was markedly turned into round by SCl. The CSA of SCI group was smaller than control group's. After sacral neuromodulation, the shape was turned into angled again. 
Nevertheless, the present study showed that sacral neuromodulation has a therapeutic potential to improve neurogenic bladder and muscle atrophy. The urodynamic study using cystometrogram revealed that the recovery of voiding contraction interval was significant in the SCI + SES group comparing to the SCI group. Furthermore, lower extremities muscle such as the gastrocnemious muscle was relatively better preserved after sacral neuromodulation.

Electrical stimulation has been reported to cause neurobiological effects, such as relieving pain in the area of head and face and restoring movement and function in individuals with spinal cord injury ${ }^{6,8)}$. It has also been indicated that electrical stimulation can directly influence on regenerating neural tissues observed under a well-controlled experimental environment $^{8)}$. Several investigators have reported that a low-frequency electrical stimulation is a promising approach to accelerate nerve regeneration after injury ${ }^{2,10}$. There are a few studies on neuroprotective effects of sacral nerve stimulation in the SCI patients ${ }^{2,3,6,9,12,14)}$. The additional effect of sacral neuromodulation was improvement of neurogenic bladder, intestinal motility, penile erection, pain discrimination, and motor power of leg'.

However, the current study showed that sacral neuromodulation to rat injured thoracic spinal cord did not improve the locomotor function. We suggest that it could be caused by an improper protocol of stimulation. Several investigators have reported that a low-frequency electrical stimulation could accelerate nerve regeneration after injury ${ }^{2,10}$. However, a high frequency of electrical stimulation may increase failure rate of nerve regeneration ${ }^{13)}$. Hence, these results cannot exclude potential beneficial effects of sacral nerve stimulation in other models of SCI with different treatment protocols, the disappointing outcomes in this study should be limited by the treatment protocol.

\section{CONCLUSION}

In a rat thoracic spinal cord contusional model, we failed to establish a rat spinal cord stimulation model. However, sacral neuromodulation has a therapeutic potential to improve neurogenic bladder and muscle atrophy. Further experimental studies are needed to establish a rat spinal cord stimulation model and to determine the most effective protocol of stimulation.

\section{ACKNOWLEDGEMENT}

This study was supported by grant No. 02-2011-052 from the Seoul National University Bundang Hospital Research Fund.
Seoul National University Bundang Hospital institutional animal care and use committee permitted this study.

\section{REFERENCES}

1. Agrawal G, Kerr C, Thakor NV, All AH: Characterization of graded multicenter animal spinal cord injury study contusion spinal cord injury using somatosensory-evoked potentials. Spine (Phila Pa 1976) 35:1122-1127, 2010

2. Al-Majed AA, Neumann CM, Brushart TM, Gordon T: Brief electrical stimulation promotes the speed and accuracy of motor axonal regeneration. J Neurosci 20:2602-2608, 2000

3. Bai C, An H, Wang S, Jiang D, Fan W, Nie H: Treatment and prevention of bacterial translocation and endotoxemia with stimulation of the sacral nerve root in a rabbit model of spinal cord injury. Spine (Phila Pa 1976) 36:363-371, 2011

4. Basso DM, Beattie MS, Bresnahan JC: A sensitive and reliable locomotor rating scale for open field testing in rats. J Neurotrauma 12:1-21, 1995

5. Basso DM, Beattie MS, Bresnahan JC: Graded histological and locomotor outcomes after spinal cord contusion using the NYU weight-drop device versus transection. Exp Neurol 139:244-256, 1996

6. Brindley GS, Polkey CE, Rushton DN, Cardozo L: Sacral anterior root stimulators for bladder control in paraplegia: the first 50 cases. J Neurol Neurosurg Psychiatry 49:1104-1114, 1986

7. Cohen DM, Patel CB, Ahobila-Vajjula P, Sundberg LM, Chacko $\mathrm{T}$, Liu SJ, et al: Blood-spinal cord barrier permeability in experimental spinal cord injury: dynamic contrast-enhanced MRI. NMR Biomed 22:332-341, 2009

8. Ebner A, Jiang C, Lindstrom S: Intravesical electrical stimulation - an experimental analysis of the mechanism of action. $\mathrm{J}$ Urol 148:920-924, 1992

9. Geremia NM, Gordon T, Brushart TM, Al-Majed AA, Verge VM: Electrical stimulation promotes sensory neuron regeneration and growth-associated gene expression. Exp Neurol 205:347359, 2007

10. Hausmann ON: Post-traumatic inflammation following spinal cord injury. Spinal Cord 41:369-378, 2003

11. Hong CH, Lee HY, Jin MH, Noh JY, Lee BH, Han SW: The effect of intravesical electrical stimulation on bladder function and synaptic neurotransmission in the rat spinal cord after spinal cord injury. BJU Int 103:1136-1141, 2009

12. Inoue M, Hojo T, Yano T, Katsumi Y: The effects of electroacupuncture on peripheral nerve regeneration in rats. Acupunct Med 21:9-17, 2003

13. Ishigooka M, Suzuki Y, Hashimoto T, Sasagawa I, Nakada T, Handa Y: A new technique for sacral nerve stimulation: a percutaneous method for urinary incontinence caused by spinal cord injury. Br J Urol 81:315-318, 1998

14. Kim KT, Nam TK, Park YS, Kim YB, Park SW: Neuroprotective effect of anthocyanin on experimental traumatic spinal cord injury. J Korean Neurosurg Soc 49:205-211, 2011

15. Lee TT, Green BA, Dietrich WD, Yezierski RP: Neuroprotective effects of basic fibroblast growth factor following spinal cord contusion injury in the rat. J Neurotrauma 16:347-356, 1999 
16. O'Gara T, Urban W, Polishchuk D, Pierre-Louis A, Stewart M: Continuous stimulation of transected distal nerves fails to prolong action potential propagation. Clin Orthop Relat Res 447: 209-213, 2006

17. Rijkhoff NJ, Wijkstra H, van Kerrebroeck PE, Debruyne FM: Selective detrusor activation by electrical sacral nerve root stimulation in spinal cord injury. J Urol 157:1504-1508, 1997

18. Sharma HS, Badgaiyan RD, Alm P, Mohanty S, Wiklund L: Neu- roprotective effects of nitric oxide synthase inhibitors in spinal cord injury-induced pathophysiology and motor functions: an experimental study in the rat. Ann N Y Acad Sci 1053:422-434, 2005

19. Sharma N, Coughlin L, Porter RG, Tanzer L, Wurster RD, Marzo SJ, et al: Effects of electrical stimulation and gonadal steroids on rat facial nerve regenerative properties. Restor Neurol Neurosci $27: 633-644,2009$ 